\title{
Optical module to extend any Fourier-domain optical coherence tomography system into a polarisation-sensitive system
}

\author{
Sylvain Rivet ${ }^{1,2}$, Manuel J. Marques ${ }^{1, *}$, Adrian Bradu ${ }^{1}$ and \\ Adrian Podoleanu ${ }^{1}$ \\ 1 Applied Optics Group, School of Physical Sciences, University of Kent, Canterbury \\ CT2 7NH, UK \\ 2 Université de Bretagne Occidentale, EA 938 Laboratoire de Spectrométrie et \\ Optique Laser, 6 avenue Le Gorgeu, C.S. 93837, 29238 Brest Cedex 3, France \\ E-mail: mjmm2@kent.ac.uk
}

December 2015

\begin{abstract}
This article presents a theoretical study on an optical module (OM) that can be inserted between an object under investigation and a Fourier-domain optical coherence tomography (FD-OCT) system, transforming the latter into a polarisationsensitive OCT (PS-OCT) system. The module consists of two electro-optic modulators, a Faraday rotator, a linear polariser and a quarter-wave plate. A detailed description on how the module can be used to extract both the net retardance and the fast axis orientation of a linear birefringent sample is presented. This is achieved by taking two sequential measurements for different values of retardance produced by the electrooptic modulator. The module keeps measurements free from undesired polarimetric effects due to birefringence in the single-mode optical fibre and diattenuation in fibrebased couplers within OCT systems. Simulations have been carried out in order to evaluate the effects of chromatic behaviour of the components within the OM.
\end{abstract}

Submitted to: J. Opt.

\section{Introduction}

Polarisation-sensitive optical coherence tomography (PS-OCT) methods emerged as early as 1992 [1], evolving from bulk-based to more compact fibre-based designs. PSOCT systems operate either in time-domain $[2,3,4]$ or in frequency-domain $[5,6,7]$.

PS-OCT systems are useful in medical OCT applications due to the link between polarisation properties and the health state of tissue. In non-destructive testing, PS-OCT also provides birefringence information, useful in assessing the mechanical properties of the structures evaluated. Due to their versatility, easy alignment, compact size [8] and also to the need for single spatial mode selection, fibre-based systems are used 
in OCT practice. However, external factors (such as temperature and mechanical stress) affect the birefringence of single-mode fibres (SMF) used in OCT systems, inducing disturbances [9] in the measured polarisation. This difficulty has been overtaken by 1) building specific OCT systems and 2) measuring (sequentially or instantaneously) the polarimetric properties of fibres so as to compensate for their effects. Yet the OCT systems dedicated to polarisation are sometimes complex and non versatile. Due to the use of optical fibres, the incident polarisation state is not a single-circular state, but this issue can be overcome by probing the sample with at least two orthogonal polarisation states $[2,7]$. Alternatively, one can take into account the polarimetric properties of the fibres by performing careful calibration steps, in this way ensuring that the probing beam is in a single, circular polarisation state [10, 11].

Another solution is to perform in free space both the generation of the polarisation states and the analysis of their modifications caused by the sample. Such an approach has been adopted in Roth et al. [12] and in Marques et al. [13]. The solution presented in [12] requires at least 3 measurements to extract the polarimetric information. In our previously reported system [13], only 2 measurements were used because the input polarisation state was always circular. However, this required splitting the beam within the sample arm, bringing further losses.

To measure the sample's polarimetric properties, we propose a novel approach, without any calibration step involving the optical fibres. This approach is based on an in-line optical module (OM) placed before the sample being evaluated. By a suitable design of the module, this can be used to convert any conventional OCT system into a PS-OCT system. A theoretical model presenting the operation of such a module is developed in Section 2. Two sequential measurements are used to provide polarisation characterisation of the birefringent sample that is independent from the polarimetric response of the SMF inside the interferometer. Simulations are carried out to evaluate how the fibre disturbances and the chromatic response of components within the OM affect the polarimetric measurements. Our simulations demonstrate that the effects due to fibre disturbances can be eliminated.

\section{Theory}

The OM (shown in figure 1 and detailed in figure 2, designed for free space operation) generates a circular polarisation state to probe the sample and to interrogate its polarimetric properties in two sequential steps. Probing the sample with circularly polarised light is a widely used technique to interrogate these properties $[1,3,4,14]$.

The optical module is inserted after the sample arm fibre of a conventional Fourierdomain OCT (FD-OCT) system, which can be a Spectrometer-based OCT (Sp-OCT) or a Swept-source-based OCT (SS-OCT). For brevity, we refer only to the Sp-OCT method, but all the theory that follows is applicable to the SS-OCT method as well.

Scanning of the sample is performed by two galvo-scanning mirrors SXY placed after the OM. Since the angles of incidence of the beam on the two mirrors in the 


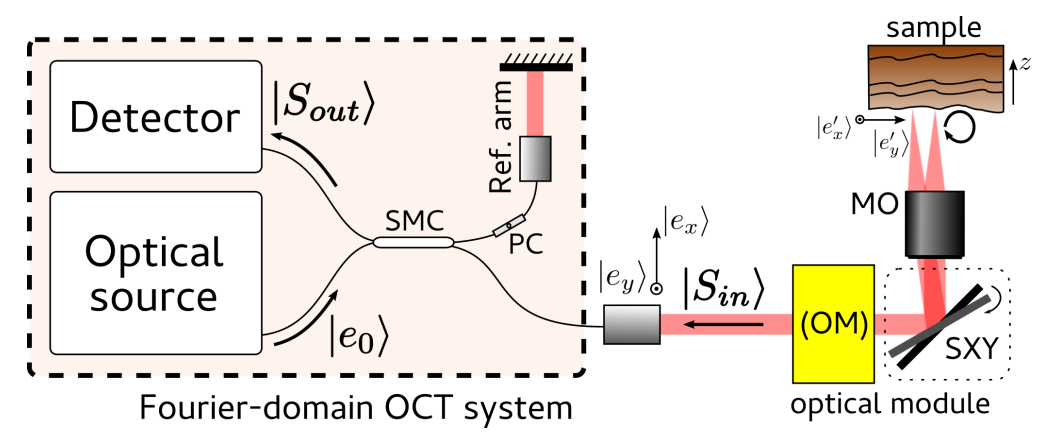

Figure 1. Fourier-domain PS-OCT system. The dashed rectangle corresponds to a fibre-based Fourier-domain OCT system. SXY, scanning head with two galvanometer scanners with axes oriented at $90^{\circ}$ in relation to each other; MO, microscope objective; PC, fibre-based polarisation controller; SMC, single-mode fibre coupler. Only singlemode optical fibres are used. The field generated by the optical source is given by $\left|e_{0}\right\rangle$. The sample is probed with a circular polarisation state. $\left|S_{i n}\right\rangle$ describes the field returning to the fibre-based part of the interferometer, and $\left|S_{\text {out }}\right\rangle$ describes the same field after propagation through the fibres and the single-mode fibre coupler.

galvanometer scanning head are relatively large (45 degrees), the Fresnel coefficients differ for perpendicular and parallel components in relation to the incidence plane of wave incident on each galvanometer scanner. By having two galvanometer scanners at 90 degrees in sequence, the Fresnel effect is cancelled, as the wave oscillating in the incident plane of the first mirror oscillates perpendicularly to the incident plane of the second mirror. Therefore, no effect on the polarisation state is considered via SXY. However if only one mirror would be used, then its effect should be taken into account, which is not the case here.

Let us consider a single reflector in the sample arm of the interferometer, placed at $z$ along the depth coordinate. The intensity $I(\nu)$ versus optical frequency $\nu$ measured at the spectrometer's camera is given by

$$
I(\nu)=I_{D C}(\nu)+A(z, \nu) e^{i \frac{2 \pi}{c} \nu 2 z}+A^{*}(z, \nu) e^{-i \frac{2 \pi}{c} \nu 2 z},
$$

where $I_{D C}(\nu)$ is the DC component of the interferometric signal, $A(z, \nu)$ is the complex amplitude of the interference between the electric field returning from the sample proportional to $\left|S_{\text {out }}\right\rangle$ and the field $\left|r_{\text {out }}(\nu)\right\rangle$ from the reference arm. $c$ is the speed of light and $z$ is the depth of the object measured from optical path difference (OPD) $=0$. The aforementioned field returning from the sample arm is given by $r(z)\left|S_{\text {out }}(z, \nu)\right\rangle$, where $r(z)$ is the complex amplitude reflection coefficient for a scattering centre located at depth $z$. In addition, the chromatic behaviour of fibres is taken into account by considering that the output fields are dependent on $\nu$. The complex amplitude $A(z, \nu)$ can be expressed as

$$
A(z, \nu)=r(z)\left\langle r_{\text {out }}(\nu) \mid S_{\text {out }}(z, \nu)\right\rangle .
$$

Throughout this theory section, the shape of the spectrum is considered to be flat along $\nu$. 
By Fourier transforming $I(\nu)$, an A-scan is obtained, ie., a complex-valued peak corresponding to the single reflector located at depth $z$. Let $\hat{A}(z)$ be the complex value at the maximum of this peak. This is related to $A(z, \nu)$ as follows:

$$
\hat{A}(z)=\int A(z, \nu) d \nu
$$

and its absolute value $\hat{I}_{\text {interf }}$ can be written as

$$
\hat{I}_{\text {interf }}(z)=|\hat{A}(z)|=\left|r(z) \int\left\langle r_{\text {out }}(\nu) \mid S_{\text {out }}(z, \nu)\right\rangle d \nu\right| .
$$

The field $\left|S_{\text {out }}(z, \nu)\right\rangle$ contains both the retardance of the sample under analysis, and the polarimetric properties of the fibres and couplers, which vary significantly with the environmental parameters. If the contributions from the sample and the fibres/couplers are decoupled, then the polarimetric response of the sample can be identified independently of that from the fibres.

\subsection{Description of the optical module (OM)}

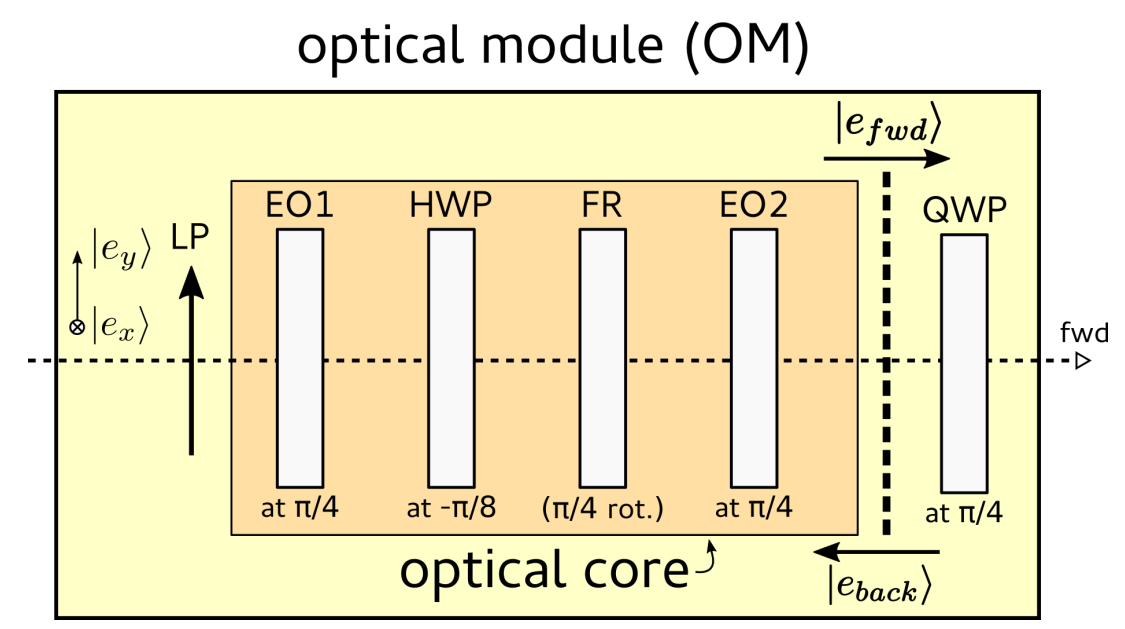

Figure 2. Optical module $(\mathrm{OM})$ to convert any FD-OCT system into a PS-FD-OCT system. LP, linear polariser oriented along $\left|e_{y}\right\rangle$ (side view of the diagram in figure 1). QWP, quarter-wave plate oriented at $\pi / 4$ in relation to $\left|e_{x}\right\rangle$. Inside the OM, another optical block is defined as the optical core of the OM. The optical core consists of two electro-optic polarisation modulators (EO1 and EO2) oriented at $\pi / 4$ according to $\left|e_{x}\right\rangle$, a half-wave plate (HWP) oriented at $-\pi / 8$, and a Faraday rotator (FR) that induces a polarisation state rotation of $\pi / 4$. The operation of the optical core is described in the main text. $\left|e_{f w d}\right\rangle$ describes the polarisation state of the light probing the sample before the quarter-wave plate (QWP) of the OM. $\left|e_{b a c k}\right\rangle$ describes the polarisation state of light returning from the sample after passing through the QWP.

The OM comprises a total of six elements, starting with a linear polariser (LP) and ending with a quarter-wave plate (QWP), ensuring in this way a circular polarisation state in front of the sample (figure 2). Performing polarimetric measurements on 

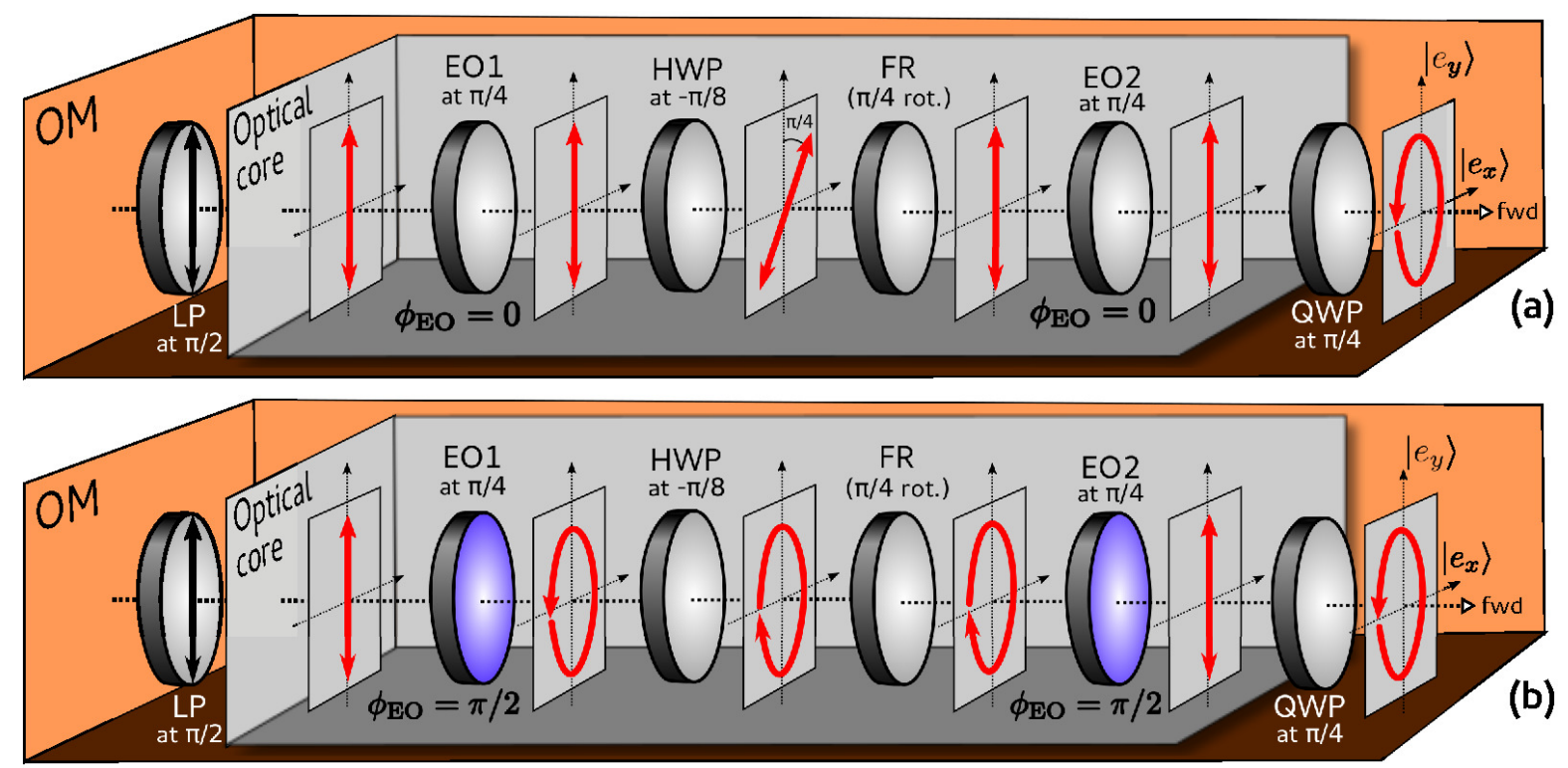

Figure 3. Orientation of the polarisation states within the OM (figure 2) for $\phi_{E O}=0$ (a) and $\phi_{E O}=\pi / 2$ (b) for forward propagation (left to right). Regardless of the value $\phi_{E O}$ of the EO's retardance, the output polarisation state is always circular.

the sample requires analysing the two orthogonal polarisation components. The LP however favours a single polarisation state. Therefore, to circumvent this limitation, an optical core comprising two electro-optic polarisation modulators (EOs), a half-wave plate (HWP) and a Faraday rotator (FR) is used according to the procedure described below. Depending on the propagation direction of the wave, forward and backward from the sample, the optical core is used to rotate the polarisation orientation by either $90^{\circ}$ or $0^{\circ}$ respectively. The different operation of the optical core does not affect the circular polarisation of the wave propagating towards the sample nor the polarisation state of the wave returned from the sample containing the polarisation properties of the sample.

Detailing the OM's behaviour further, linearly polarised light after the LP is oriented along $\left|e_{y}\right\rangle$ and propagates through an optical core comprising an electrooptic polarisation modulator (EO1) oriented at $\pi / 4$, a $\mathrm{HWP}$ oriented at $-\pi / 8$, a FR which rotates the incident polarisation by $\pi / 4$, and a second electro-optic polarisation modulator (EO2) oriented at $\pi / 4$. EO1 and EO2 are driven to exhibit the same retardance $\phi_{E O}$. The orientation of their fast axes is defined in respect to $\left|e_{x}\right\rangle$ in the basis $\left\{\left|e_{x}\right\rangle,\left|e_{y}\right\rangle\right\}$.

In order to visualise the change of the polarisation states induced by the optical core in forward propagation, the retardance $\phi_{E O}$ is set to 0 or $\pi / 2$, as shown in figure 3 .

For the field going forward through the optical core (figure 3), there is no change in the polarisation state of light and this result can be generalized irrespective of the values of $\phi_{E O}$ introduced by EO1 and EO2. Indeed, the Jones matrix of the optical core 
in forward direction, $J_{\text {core }}^{f w d}$, is

$$
J_{\text {core }}^{f w d}\left(\phi_{E O}\right)=-i\left(\begin{array}{cc}
-1 & 0 \\
0 & 1
\end{array}\right)
$$

Therefore the field $\left|e_{f w d}\right\rangle=J_{\text {core }}^{f w d}\left|e_{y}\right\rangle=-i\left|e_{y}\right\rangle$ after EO2, and is linearly polarised along $\left|e_{y}\right\rangle$. Then the electric field is converted into a circular polarisation state by the quarter-wave plate oriented at $45^{\circ}$. The sample is modelled as a linear retarder with a net (or double-pass) retardance $2 \varphi(z)$, and a fast axis orientation given by $\theta(z)$ in respect to the sample basis $\left\{\left|e_{x}^{\prime}\right\rangle,\left|e_{y}^{\prime}\right\rangle\right\}$, as defined in figure 1 . This basis results from the transformation of the original $\left\{\left|e_{x}\right\rangle,\left|e_{y}\right\rangle\right\}$ basis by the reflections in the two galvo-scanners SXY.

Light back-reflected by the birefringent sample travels towards the QWP of the OM. The corresponding field $\left|e_{\text {back }}\right\rangle$ (figure 2) can be expressed on the vector basis $\left\{\left|e_{x}\right\rangle,\left|e_{y}\right\rangle\right\}$ as follows (see Appendix B):

$$
\left|e_{\text {back }}\right\rangle=\cos \varphi(z)\left|e_{x}\right\rangle-\sin \varphi(z) e^{-2 i \theta(z)}\left|e_{y}\right\rangle
$$

The range for the one-pass retardance $\varphi(z)$ is considered from 0 to $\pi / 2$, while the range for the fast axis orientation $\theta(z)$ is measured between 0 and $\pi$.

In the backward direction, the optical core modifies the polarisation state according to the retardance $\phi_{E O}$ imprinted by the two EOs. In particular, the core acts as a rotator of the polarisation state as shown in figure 4 for $\phi_{E O}$ set at 0 (a) or $\pi / 2$ (b).

For any retardance $\phi_{E O}$ applied identically on both EOs, the Jones matrix describing the optical module in backward direction can be written on the basis $\left\{\left|e_{x}\right\rangle,\left|e_{y}\right\rangle\right\}$ as

$$
J_{\text {core }}^{\text {back }}\left(\phi_{E O}\right)=-i\left(\begin{array}{cc}
i \sin \phi_{E O} & \cos \phi_{E O} \\
\cos \phi_{E O} & i \sin \phi_{E O}
\end{array}\right)
$$

After traversing the optical core, the beam passes through the linear polariser, oriented along $\left|e_{y}\right\rangle$, with the resulting field $\left|S_{i n}(z)\right\rangle$ at the input of the probe fibre, as shown in figure 1. According to the retardance $\phi_{E O}$ of both EOs, this field becomes

$$
\left|S_{\text {in }}(z)\right\rangle=P \cdot J_{\text {core }}^{\text {back }}\left(\phi_{E O}\right) \cdot\left|e_{\text {back }}\right\rangle,
$$

where $P$ is the Jones matrix of the linear polariser oriented along $\left|e_{y}\right\rangle$ described by

$$
P=\left(\begin{array}{ll}
0 & 0 \\
0 & 1
\end{array}\right)
$$

Then the field $\left|S_{i n}(z)\right\rangle$ can be expressed according to

$$
\left|S_{i n}(z)\right\rangle=-\left(i \cos \varphi(z) \cos \phi_{E O}+e^{-2 i \theta(z)} \sin \varphi(z) \sin \phi_{E O}\right)\left|e_{y}\right\rangle .
$$

Due to the polarimetric response of the collecting fibre and of the coupler described in the general matrix $J_{\text {out }}^{\text {fibre }}(\nu)$, the field $\left|S_{\text {out }}(z, \nu)\right\rangle$ in front of the detector is obtained as a transformation of $\left|S_{i n}\right\rangle$, according to: 

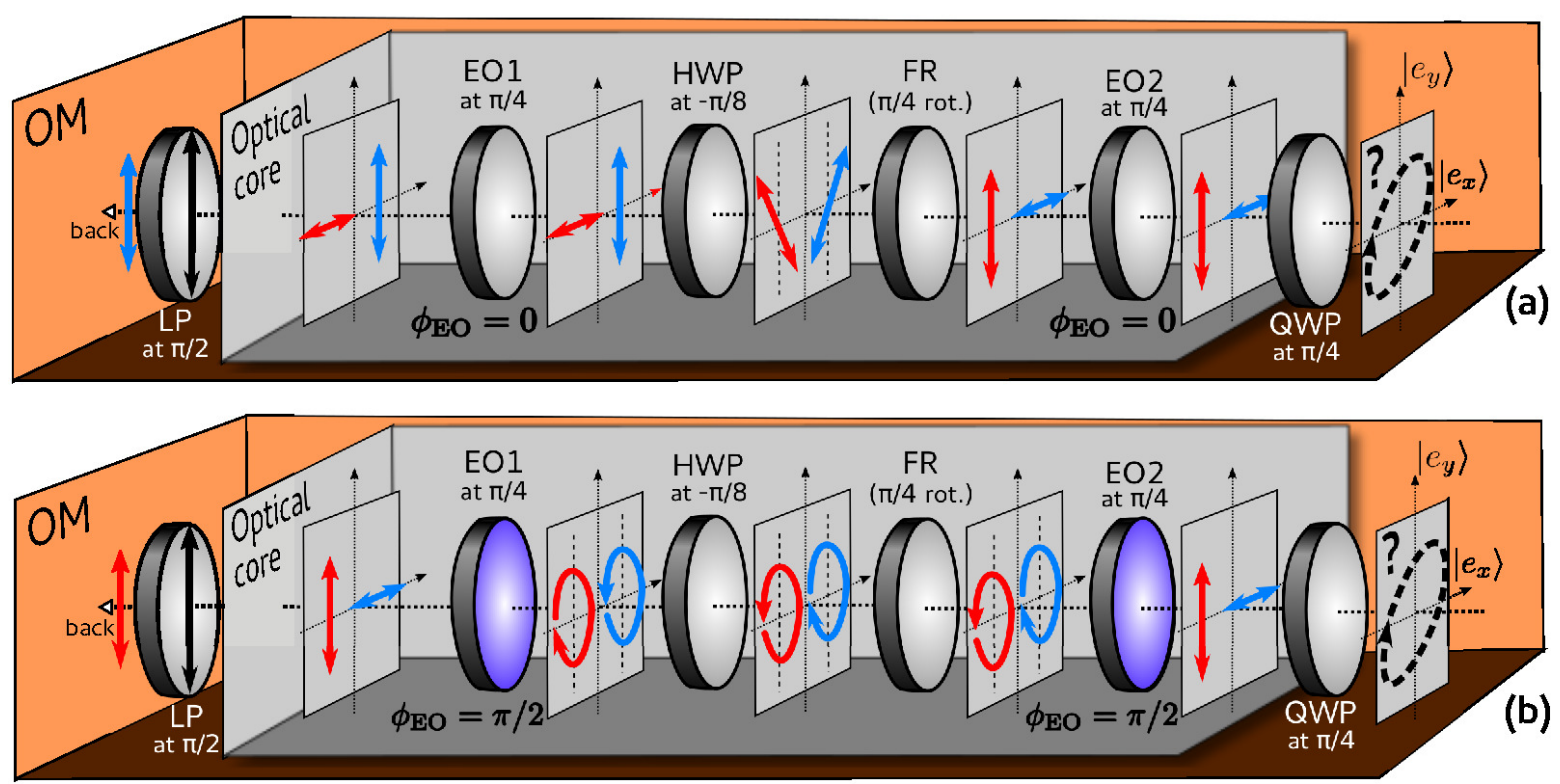

Figure 4. Orientation of polarisation states in the $\mathrm{OM}$ for $\phi_{E O}=0$ (a) and $\phi_{E O}=\pi / 2$ (b) for the backward propagation (right to left). The probing beam returns from the sample with an unknown polarisation state, which is composed of two linear orthogonal components, represented as red and blue in the diagram. These are sequentially selected, depending on the value of $\phi_{E O}$. The two orthogonal components are represented in the diagram as being spatially separated for clarity.

$$
\begin{aligned}
\left|S_{\text {out }}(z, \nu)\right\rangle= & J_{\text {out }}^{\mathrm{fibre}}(\nu)\left|S_{\text {in }}\right\rangle \\
= & -\left(i \cos \varphi(z) \cos \phi_{E O}\right. \\
& \left.+e^{-2 i \theta(z)} \sin \varphi(z) \sin \phi_{E O}\right)\left|e_{\text {out }}(\nu)\right\rangle,
\end{aligned}
$$

where $\left|e_{\text {out }}(\nu)\right\rangle=J_{\text {out }}^{\text {fibre }}(\nu)\left|e_{y}\right\rangle$. Then the complex amplitude $\hat{A}^{\phi_{E O}}(z)$, for the A-scan peak corresponding to the depth $z$ of the scattering centre, can be written from (2) and (3) as

$$
\hat{A}^{\phi_{E O}}(z)=-\alpha(z)\left(i \cos \varphi(z) \cos \phi_{E O}+e^{-2 i \theta(z)} \sin \varphi(z) \sin \phi_{E O}\right) e^{i \varphi_{\text {rand }}},(13
$$

where the random phase $\varphi_{\text {rand }}$ is added to take into account the noise of the interferometer (caused by random changes in the $\mathrm{OPD}$ ), and $\alpha(z)$ is defined by

$$
\alpha(z)=r(z) e^{i \pi} \int\left\langle r_{\text {out }}(\nu) \mid e_{\text {out }}(\nu)\right\rangle d \nu .
$$

To extract the polarimetric information, $\varphi$ and $\theta$, irrespective of the polarimetric properties of the fibres in the interferometer, $\hat{A}(z)$ needs to be measured sequentially for two different values of $\phi_{E O}$. Since the linear polariser is used as an analyser in the backward direction, the two polarisation states of the light returning from the sample and travelling through the fibres for the two values of $\phi_{E O}$ are identical. It means that the coefficient $\alpha(z)$, which contains all the polarimetric disturbances of the fibres, is 
constant and can be eliminated by using a similar procedure to that described in our previous paper [13]. Furthermore, the product $\left\langle r_{\text {out }}(\nu) \mid e_{\text {out }}(\nu)\right\rangle$ dictates the contrast of the interference signal, i.e. the signal-to-noise ratio of the OCT signal. This can be optimised by adjusting the fibre-based polarisation controller PC present in the reference arm as pictured in figure 1. Since $\alpha(z)$ is eliminated in the procedure mentioned above, the adjustment of PC does not impact in any way the polarimetric measurement.

We present two case studies for different switching rates of the EOs in comparison with the variation of the random phase $\varphi_{\text {rand }}$ in the system.

\subsection{Fast set-up: Random phase $\varphi_{\text {rand }}$ identical for the two sequential measurements}

If the sequential measurements are carried out with a high switching rate of the EOs, the variation of $\varphi_{\text {rand }}$ can be considered negligible from one measurement to the next. Let us define a function $\beta(z)$ to consider the two sequential measurements, one for $\phi_{E O}=\phi_{0}$ and the other for $\phi_{E O}=\phi_{0}+\pi / 2$, as follows

$$
\beta(z)=\frac{\sin \phi_{0} \hat{A}^{\phi_{0}}(z)+\cos \phi_{0} \hat{A}^{\phi_{0}+\pi / 2}(z)}{\cos \phi_{0} \hat{A}^{\phi_{0}}(z)-\sin \phi_{0} \hat{A}^{\phi_{0}+\pi / 2}(z)} .
$$

By using the expressions of $\hat{A}^{\phi_{E O}}$ in $(13), \beta(z)$ can be written according to the polarimetric properties of the sample as

$$
\beta(z)=\tan \varphi(z) e^{-i(2 \theta+\pi / 2)} .
$$

The function $\beta(z)$ is therefore insensitive to the polarimetric properties of the fibres and couplers contained in $\alpha(z)$. From (16), the value of the one-pass retardance of the sample, $\varphi(z)$, is given by

$$
\varphi(z)=\arctan |\beta(z)|,
$$

with $\varphi(z) \in[0, \pi / 2]$ and the fast axis orientation $\theta(z)$ of the sample given by

$$
\theta(z)=-\arg [\beta(z)] / 2-\pi / 4+m \pi
$$

where arg stands for the argument of the complex number in the bracket and $m$ is an integer such that $\theta(z) \in[0, \pi]$.

As a result of the above demonstration, the measurement of two complex amplitudes for two $\phi_{E O}$ values $\left(\phi_{0}\right.$ and $\left.\phi_{0}+\pi / 2\right)$ is enough to obtain the retardance and the orientation of the optical axis of the retarder. By calculating $\beta(z)$, the coefficient $\alpha(z)$ incorporating the fibre polarimetric properties is eliminated, therefore the retardance $\varphi(z)$ and the fast axis orientation $\theta(z)$ are independent from these properties. 


\subsection{Slow set-up: Random phase $\varphi_{\text {rand }}$ not identical for the two sequential} measurements

As the sequential measurements are not carried out sufficiently fast to ignore the random phase variation, a different random phase must be taken into consideration for each of the two sequential measurements. This could be the case if the EOs are switched after acquiring each 2D image (B-scan).

If we are only interested in the net retardance, we propose measuring the complex amplitude for $\phi_{E O}=0$ and $\phi_{E O}=\pi / 2$. Indeed, $\varphi(z)$ can be extracted irrespective of the random phase due to

$$
\varphi(z)=\arctan \left|\frac{\hat{A}^{\pi / 2}(z)}{\hat{A}^{0}(z)}\right| .
$$

If we need to measure the axis orientation of the sample in addition to its retardance, then a possible solution consists in using the surface layer of the sample to produce a phase reference. Usually the surface layer of the medium is used in PS-OCT to reference the polarimetric properties of the fibre $[5,7]$ corresponding to a normalisation in relation to the surface. The coding and decoding is performed in free space, in front of the sample. This allows independence of measurements from the fibre's polarimetric properties [13]. To eliminate the random phase, we need to calculate the following ratio

$$
\hat{A}_{\text {norm }}^{\phi_{E O}}(z)=\frac{\hat{A}^{\phi_{E O}}(z)}{\hat{A}^{\phi_{E O}}(0)},
$$

where $\hat{A}^{\phi_{E O}}(0)$ is the complex amplitude for the A-scan peak at the surface, the value of which depends on the electro-optic retardance $\phi_{E O}$.

The choice of the values for $\phi_{E O}$ depends on whether a retarder element is placed in front of the surface or not (figure 5). The required values for $\phi_{E O}$ are listed in table 1 for each of the cases studied below.

2.3.1. No retarder element in front of the sample surface, $\varphi(0)=0$. When there is no retarder element in front of the surface (e.g. skin), the net retardance imprinted by the surface is considered to be $\varphi(0)=0$. The complex amplitude is therefore $\hat{A}^{\pi / 2}(0)=0$

\begin{tabular}{|c|c|c|}
\cline { 2 - 3 } \multicolumn{1}{c|}{} & $\Delta \varphi_{\text {rand }}=0$ & $\Delta \varphi_{\text {rand }} \neq 0$ \\
\hline$\varphi(0)=0$ & & $\phi_{E O}: \pi / 4 \rightarrow-\pi / 4$. \\
$\varphi(0)=\pi / 2$ & $\phi_{E O}: \phi_{0} \rightarrow \phi_{0}+\pi / 2$. & $\phi_{E O}: \pi / 4 \rightarrow-\pi / 4$. \\
$\varphi(0) \neq 0, \varphi(0) \neq \pi / 2$ & & $\phi_{E O}: 0 \rightarrow \pi / 2$. \\
\hline
\end{tabular}

Table 1. Required retardance values $\phi_{E O}$ for the two EOs according to the retardance at the sample surface $\varphi(0)$ and the variation of the random phase $\Delta \varphi_{\text {rand }}$ between two switches of the EOs. 
and it cannot be used to normalise $\hat{A}^{\pi / 2}(z)$. Hence, we propose measuring the complex amplitude for $\phi_{E O}=-\pi / 4$ and $\phi_{E O}=\pi / 4$, and studying the function $\beta_{1}(z)$ defined by

$$
\beta_{1}(z)=\frac{\hat{A}_{\text {norm }}^{\pi / 4}(z)-\hat{A}_{\text {norm }}^{-\pi / 4}(z)}{\hat{A}_{\text {norm }}^{\pi / 4}(z)+\hat{A}_{\text {norm }}^{-\pi / 4}(z)}=\tan \varphi(z) e^{-i(2 \theta(z)+\pi / 2)},
$$

where $\varphi(z)$ can be retrieved using

$$
\varphi(z)=\arctan \left|\beta_{1}(z)\right|,
$$

and where the axis orientation $\theta(z)$ of the sample is given by

$$
\theta(z)=-\arg \left[\beta_{1}(z)\right] / 2-\pi / 4+m \pi .
$$

2.3.2. Retarder element in front of the sample surface, $\varphi(0) \neq 0$. An important application case is that of polarisation characterisation of the retina, where the measurement is affected by the birefringence of the anterior chamber structures in the eye. We refer to this case as that of a retarder element in front of the surface, where the retardance $\varphi(0) \neq 0$ for the surface.

If $\varphi(0) \neq \pi / 2$, we have $\hat{A}^{0}(0) \neq 0$ and $\hat{A}^{\pi / 2}(0) \neq 0$ for the surface, and they can be used to normalise the complex amplitudes $\hat{A}^{0}$ and $\hat{A}^{\pi / 2}(z)$. In such cases we propose to measure the complex amplitude for $\phi_{E O}=0$ and $\phi_{E O}=\pi / 2$. According to (19), the one-pass retardance of the sample+retarder system $\varphi(z)$ can be calculated, while the optical axis $\theta(z)$ of the medium can be expressed as

$$
\theta(z)-\theta(0)=-\arg \left[\frac{\hat{A}_{\text {norm }}^{\pi / 2}(z)}{\hat{A}_{\text {norm }}^{0}(z)}\right] / 2+m \pi .
$$

It is not possible in this case to measure the absolute orientation of the optical axis, but only the orientation according to $\theta(0)$.

(a)

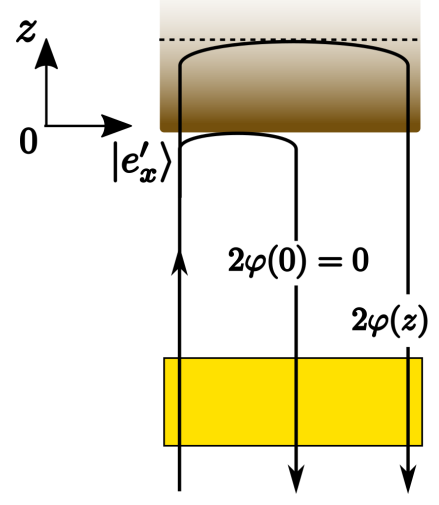

(b)

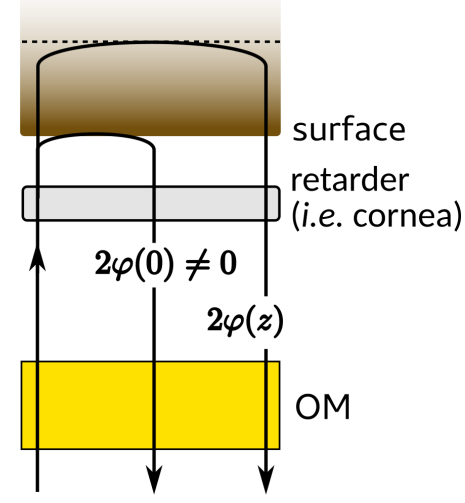

Figure 5. Representation of two situations: (a) no retarder in front of the surface, (b) retarder in front of the surface, which as an example can be taken as corneal tissue. The sample is considered without diattenuation. 
If $\varphi(0)=\pi / 2$, the complex amplitude $\hat{A}^{0}(0)$ is null and it is not possible to use it to normalise $\hat{A}^{0}(z)$. We therefore propose measuring the complex amplitude for $\phi_{E O}=-\pi / 4$ and $\phi_{E O}=\pi / 4$, and studying the function $\beta_{2}(z)$ defined by

$$
\beta_{2}(z)=\frac{\hat{A}_{\text {norm }}^{\pi / 4}+\hat{A}_{\text {norm }}^{-\pi / 4}}{\hat{A}_{\text {norm }}^{\pi / 4}-\hat{A}_{\text {norm }}^{-\pi / 4}}=\tan \varphi(z) e^{-i(2 \theta(z)+\pi / 2)},
$$

where $\varphi(z)$ can be retrieved from

$$
\varphi(z)=\arctan \left|\beta_{2}(z)\right|
$$

and the axis orientation $\theta(z)$ by

$$
\theta(z)=-\arg \left[\beta_{2}(z)\right] / 2-\pi / 4+m \pi
$$

\section{Simulations}

The OCT system is modelled with a Gaussian broadband source at $\lambda_{0}=850 \mathrm{~nm}$ for two situations: full width at half maximum (FWHM) $\Delta \lambda=100 \mathrm{~nm}$ and $\Delta \lambda=50 \mathrm{~nm}$. The channelled spectrum is re-sampled to eliminate the chirp effect due to the dispersion of the OCT system, and the non-linearities in wavenumber present in the spectrometer. In each case, the channelled spectrum is sampled with 1000 points. The span of the spectrometer is equal to the FWHM of the source. The polarimetric response of the fibres is modelled by the product $J^{\text {Fibre }}$ of different Jones matrices, accounting for the chromatic birefringence of the fibre and the possible diattenuation of the couplers, as follows

$$
J^{\text {Fibre }}=J_{\operatorname{Rot}(\alpha 1)} \cdot J_{\operatorname{Diat}(D, \alpha 2)} \cdot J_{\operatorname{Bir}(\phi(\nu), \alpha 3)} \cdot J_{\operatorname{Rot}(\alpha 4)},
$$

where $J_{R o t(\alpha)}$ is the Jones matrix for a rotator that rotates the linear polarisation by an angle $\alpha, J_{\operatorname{Diat}(D, \alpha)}$ corresponds to a linear diattenuator with the diattenuation $D$ and oriented at the angle $\alpha$ and $J_{\operatorname{Bir}(\phi(\nu), \alpha)}$ corresponds to a linear retarder oriented at the angle $\alpha$ with retardance $\phi(\nu)$. In this simulation, the retardance $\phi(\nu)$ is expressed according to a mean retardance $\phi\left(\nu_{0}\right)$ and a parameter $\Delta T$ to take into account the polarisation mode dispersion (PMD) of fibres, as follows

$$
\phi(\nu)=\phi\left(\nu_{0}\right)+2 \pi \Delta T\left(\nu-\nu_{0}\right)
$$

with $\nu_{0}$ being the central optical frequency of the source.

The field $\left|S_{\text {out }}(z, \nu)\right\rangle$ coming from the sample and travelling through fibres is expressed as follows

$$
\left|S_{\text {out }}(z, \nu)\right\rangle=J_{\text {out }(\nu)}^{\text {Fibre }}\left|S_{\text {in }}(z, \nu)\right\rangle,
$$

with

$$
\left|S_{i n}\right\rangle=J_{\text {back }\left(\nu, \phi_{E O}\right)}^{O M} \cdot J_{\text {sample }(z)} \cdot J_{f w d\left(\nu, \phi_{E O}\right)}^{O M} \cdot J_{\text {in }(\nu)}^{\text {Fibre }}\left|e_{0}\right\rangle
$$



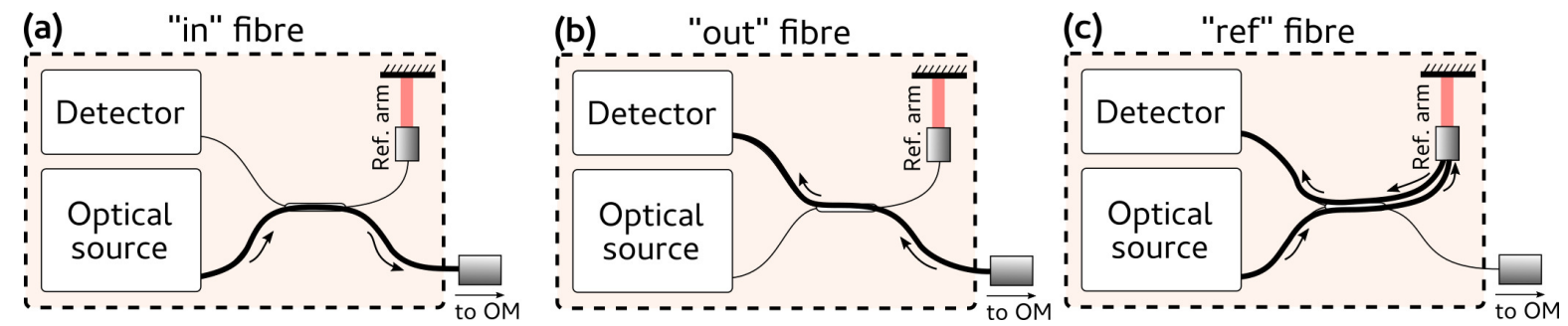

Figure 6. Schematic representation of the different fibre paths employed in the simulations.

where $\left|e_{0}\right\rangle$ is the input field from the source, $J_{\text {sample }(z)}$ is the Jones matrix of the sample after a round-trip in depth $z, J_{\text {in }(\nu)}^{\text {Fibre }}$ and $J_{\text {out }(\nu)}^{\text {Fibre }}$ respectively correspond to the polarisation changes of the light traveling from the source to the OM and from the optical module to the detector, $J_{f w d\left(\nu, \phi_{E O}\right)}^{O M}$ and $J_{b a c k\left(\nu, \phi_{E O}\right)}^{O M}$ correspond to the polarimetric response of the $\mathrm{OM}$ in forward and backward directions defined by

$$
J_{f w d\left(\nu, \phi_{E O}\right)}^{O M}=J_{B i r(\pi / 2,-\pi / 4)} \cdot J_{\text {core }\left(\nu, \phi_{E O}\right)}^{f w d} \cdot P,
$$

and

$$
J_{b a c k\left(\nu, \phi_{E O}\right)}^{O M}=P \cdot J_{\text {core }\left(\nu, \phi_{E O}\right)}^{\text {back }} \cdot J_{B i r(\pi / 2, \pi / 4)},
$$

with $P$ being the Jones matrix of the linear polariser in the OM. The field $\left|r_{\text {out }}(\nu)\right\rangle$ coming from the reference arm is expressed with the Jones matrix of the reference fibre $J_{\text {ref }}^{\text {Fibre }}$, and a complex exponential term $e^{i \frac{2 \pi \nu}{c} 2 z}$, considering the OPD between the two arms $(2 z)$ as follows

$$
\left|r_{\text {out }}(\nu)\right\rangle=e^{i \frac{2 \pi \nu}{c} 2 z} J_{\text {ref }}^{F i b r e}\left|e_{0}\right\rangle .
$$

The simulation consists of two parts. Firstly, the Fourier transform of the intensity $S(\nu)\left(\left\langle S_{\text {out }}(z, \nu) \mid r_{\text {out }}(\nu)\right\rangle+\left\langle r_{\text {out }}(\nu) \mid S_{\text {out }}(z, \nu)\right\rangle\right)$, is calculated, where $S(\nu)$ is the power spectrum of the light source. Secondly, the complex amplitude associated with the depth $z$ in the sample is measured at the maximum of the peak.

Let "in" fibre and "out" fibre denote the optical paths within the sample fibre, from the source to the $\mathrm{OM}$ and from the OM to the detector respectively, as presented in figure 6(a)-(b). "Ref" fibre is represented in figure 6(c), which corresponds to the optical path from the source to the detector through the reference arm. Table 2 gives the parameters of the fibres used in the simulation. Here, PMD is considered as $0.1 \mathrm{ps} / \sqrt{\mathrm{km}}$ for a single mode fibre, which for a $10 \mathrm{~m}$ fibre corresponds to a delay of $\Delta T=10 \mathrm{fs}$, used in (29). The values of the coefficients in table 2 are arbitrarily chosen, and some parameters $\left(D_{\text {out }}, \Delta T_{\text {out }}\right.$ and $\left.\phi\left(\nu_{0}\right)_{\text {out }}\right)$ in the "out" fibre are independently varied in the next section to prove the insensitivity of the retardance and optical axis orientation measurements to the polarimetric response of fibres and couplers. 


\begin{tabular}{ccccccccc}
\hline & Length $(\mathbf{m})$ & $\mathbf{D}$ & $\phi\left(\nu_{0}\right)(\mathbf{r a d})$ & $\Delta T(\mathbf{f s})$ & $\alpha_{\mathbf{1}}(\mathbf{r a d})$ & $\alpha_{\mathbf{2}}(\mathbf{r a d})$ & $\alpha_{\mathbf{3}}(\mathbf{r a d})$ & $\alpha_{\mathbf{4}}(\mathbf{r a d})$ \\
\hline "in" fibre & 10 & 0 & $\pi / 5$ & 10 & $\pi / 8$ & 1 & $-\pi / 8$ & 0 \\
"out" fibre & 10 & $0, D_{\text {out }}$ & $\pi / 8, \phi\left(\nu_{0}\right)_{\text {out }}$ & $10, \Delta T_{\text {out }}$ & 0 & 0.5 & $\pi / 12$ & $\pi / 2$ \\
"ref" fibre & 20 & 0 & $\pi / 4$ & 14 & $\pi / 4$ & 0 & $\pi / 4$ & $\pi / 4$ \\
\hline
\end{tabular}

Table 2. Coefficients for the fibres in the OCT system. PMD is considered to be equal to $0.1 \mathrm{ps} / \sqrt{\mathrm{km}}$ for a single mode fibre, which corresponds to a delay of $\Delta T=10$ fs for a $10 \mathrm{~m}$ fibre. Coefficients $D, \phi\left(\nu_{0}\right), \alpha_{1}, \alpha_{2}, \alpha_{3}$ and $\alpha_{4}$ are arbitrarily chosen. $D_{\text {out }}$, $\phi\left(\nu_{0}\right)_{\text {out }}$ and $\Delta T_{\text {out }}$ are independently varied in the simulations to assess the effect of each one in the polarimetric measurements (refer to figure 7).

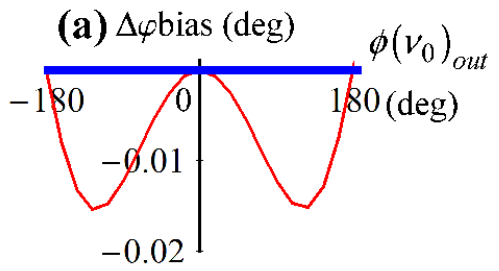

(b) $\Delta \theta$ bias (deg)

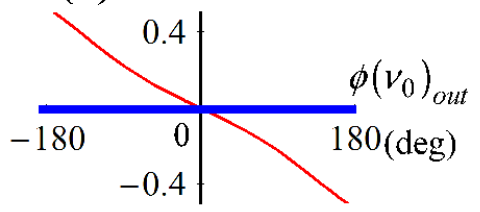

(c) $\Delta \varphi$ bias (deg)

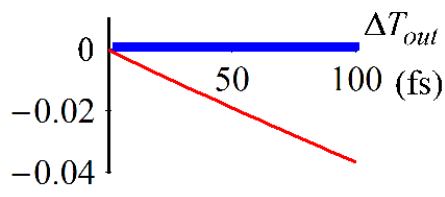

(d) $\Delta$ bias (deg)

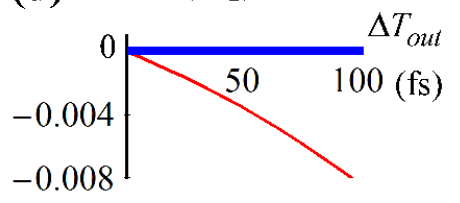

(e) $\Delta \varphi$ bias (deg)

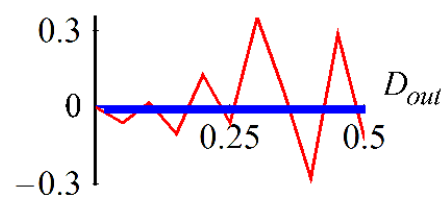

(f) $\Delta \theta \operatorname{bias}(\mathrm{deg})$

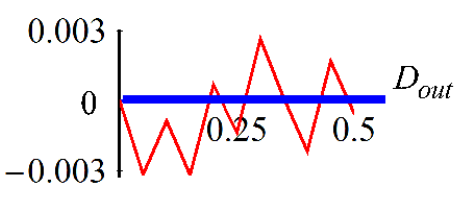

Figure 7. Measurement of the bias on the retardance $\varphi_{\text {sample }}$ and on the orientation angle $\theta_{\text {sample }}$ of a sample, according to the retardance of the "out" fibre (a) and (b), the PMD of the "out" fibre (c) and (d), and the diattenuation of the "out" fibre (e) and (f). Thick (blue) lines: the characteristics of the "out" fibre are identical for the two sequential measurements. (a) and (b) thin (red) lines: the retardance of the "out" fibre is $\phi\left(\nu_{0}\right)_{\text {out }}$ during the first measurement, $1.01 \times \phi\left(\nu_{0}\right)_{\text {out }}$ during the second measurement. (c) and (d) thin lines: the PMD of the "out" fibre is $\Delta T_{\text {out }}$ during the first measurement, $1.01 \times \Delta T_{\text {out }}$ for the second one. (e) and (f) thin lines: the diattenuation of the "out" fibre is $D_{\text {out }}$ during the first measurement, $1.01 \times D_{\text {out }}$ during the second measurement.

\subsection{Effect of fibres on the polarimetric measurement of the sample}

In the theoretical section we demonstrated that the polarimetric measurement of the sample is insensitive to fibre disturbances, since the generation and analysis of the polarisation states are done in free space, in front of the sample.

To verify this behaviour, a retarder sample oriented at $45^{\circ}$ with a retardance of $10^{\circ}$ is considered. The chromaticity of the elements in the optical module is not considered at this stage. The random phase is assumed identical for the two sequential measurements (as presented in case study 1 in section 2.2). Finally, the FWHM of the source spectrum is equal to $100 \mathrm{~nm}$ centred at $850 \mathrm{~nm}$.

The OM switches from $\phi_{E O}=0$ to $\phi_{E O}=\pi / 2$ and, according to (15), the retardance 
and the axis orientation are measured via (17) and (18). Figure 7 shows the bias of the retardance and the axis orientation by varying the properties of the "out" fibre of the sample arm.

If we consider that the fibre characteristics are identical for the two sequential measurements, the biases on the retardance $\Delta \varphi_{\text {bias }}$ and on the axis orientation $\Delta \theta_{\text {bias }}$ are equal to zero regardless of the polarimetric properties of the "out" fibre, i.e. its retardance $\phi\left(\nu_{0}\right)_{\text {out }}$ (thick blue line in figures $7(\mathrm{a})$ and $7(\mathrm{~b})$ ), its PMD $\Delta T_{\text {out }}$ (thick blue line in figures $7(\mathrm{c})$ and $7(\mathrm{~d})$ ), and its partial diattenuation $D_{\text {out }}$ (thick blue line in figures $7(\mathrm{e})$ and $7(\mathrm{f}))$. It is commonly assumed in PS-OCT that fibres remain stable during sequential measurements $[15,16]$.

We can evaluate the behaviour of the biases on retardance and axis orientation if the "out" fibre evolves between the two measurements. To do so, the parameters $\phi_{0, \text { out }}$, $\Delta T_{\text {out }}$ and $D_{\text {out }}$ are increased by $1 \%$ during the switch of the electro-optics. The results are shown as thin red lines in figure 7 . In addition to these weak biases $\left(<1^{\circ}\right)$, the chromaticity of the elements in the OM introduces other biases, as discussed in section 3.2 .

\subsection{Effect of chromatic response of the $O M$ on the polarimetric measurement of the sample}

The optical module is made of commonly available devices (Faraday rotator, wave plates, electro-optic polarisation modulators, linear polariser), similar to those widely used in PS-OCT and in interferometric systems. While achromatic wave plates and linear polarisers are readily available, the chromaticity of Faraday rotators and electro-optical polarisation modulators has to be taken into account. Consequently, systematic errors (bias) on the polarimetric measurement of the sample are induced.
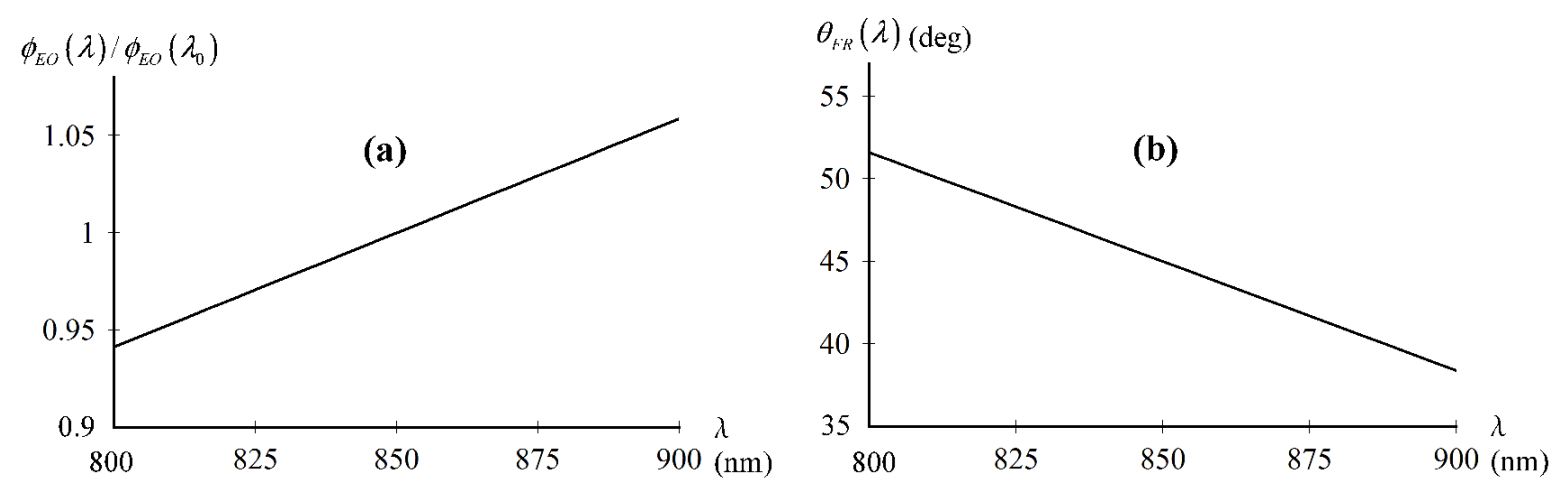

Figure 8. Chromatic response of the electro-optic polarisation modulator (a) and the Faraday rotator (b).

Electro-optic polarisation modulators are usually built with two matched crystals arranged in series, oriented at $90^{\circ}$ relative to each other, in order to suppress intrinsic birefringence and variations due to temperature changes. The simulation has considered 

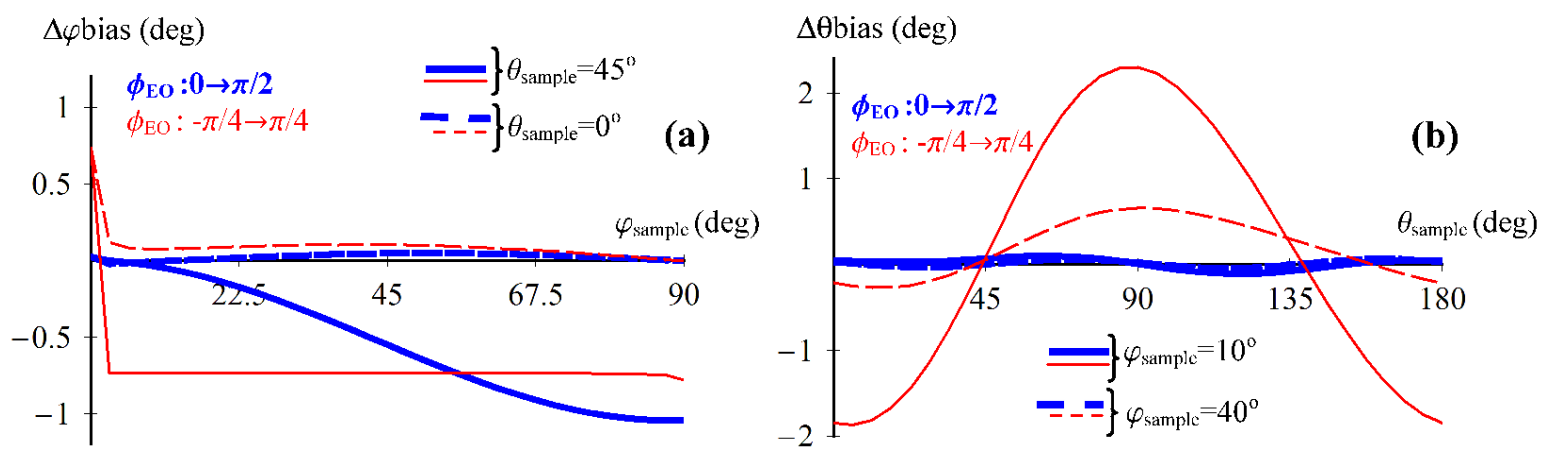

Figure 9. Measurement of the bias on the retardance $\varphi_{\text {sample }}$ (a) and the orientation angle $\theta_{\text {sample }}(\mathrm{b})$ of a linear retarder. Thin curves, retardance $\phi_{E O}$ switching from $-\pi / 4$ to $\pi / 4$. Thick curves, retardance $\phi_{E O}$ switching from 0 to $\pi / 2$. Solid lines in (a), sample fast axis oriented at $45^{\circ}$. Dashed lines in (a), sample fast axis oriented at $0^{\circ}$. Solid lines in (b), sample retardance equal to $10^{\circ}$. Dashed lines in (b), sample retardance equal to $40^{\circ}$.

a Lithium Niobate medium with a half-wave voltage $V_{\pi}$ equal to $300 \mathrm{~V}$ at $1000 \mathrm{~nm}$ and $150 \mathrm{~V}$ at $532 \mathrm{~nm}$ (such as the Newport model 4104 which has been employed in the system reported in [17]). Figure 8(a) shows the normalised retardance variation $\phi_{E O}(\lambda) / \phi_{E O}\left(\lambda_{0}\right)$ of the electro-optic polarisation modulator versus wavelength used in the simulation, with $\lambda_{0}=850 \mathrm{~nm}$.

Figure $8(\mathrm{~b})$ shows the rotation $\theta_{F R}(\lambda)$ of a $45^{\circ}$ Terbium Gallium Garnet Faraday rotator versus wavelength, which was used in the simulation. To model this, the Verdet constant $V(\lambda)$ of the rotator was linearly interpolated using values from [18], which give $\mathrm{V}$ as $51 \mathrm{rad} / \mathrm{Tm}$ for $900 \mathrm{~nm}$ and $68.5 \mathrm{rad} / \mathrm{Tm}$ for $800 \mathrm{~nm}$.

3.2.1. Bias for the fast set-up case: random phase $\varphi_{\text {rand }}$ identical for the two sequential measurements. In this section we consider that during the two sequential measurements the random phase is identical and fibres do not change their characteristics. The sample is a linear retarder where its retardance $\varphi_{\text {sample }}$ and its axis orientation $\theta_{\text {sample }}$ are variable parameters. Let us also consider the FWHM of the source spectrum to be $\Delta \lambda=100$ $\mathrm{nm}$, centred at $\lambda_{0}=850 \mathrm{~nm}$.

Biases on retardance and axis orientation are evaluated according to the values of $\varphi_{\text {sample }}, \theta_{\text {sample }}$ and the choice of retardance of the electro-optic polarisation modulators (table 1). In particular the study is carried out for two configurations to evaluate the impact of the parameter $\phi_{0}$ on the biases: 1) sequential measurements, one for $\phi_{E O}=0$ and the other one for $\phi_{E O}=\pi / 2$ (thick blue curves in figure 9 ), 2) sequential measurements, one for $\phi_{E O}=-\pi / 4$ and one for $\phi_{E O}=\pi / 4$ (thin red curves in figure $9)$.

Figure 9 (a) shows the bias on retardance for two different orientations of the retarders, at $0^{\circ}$ (dashed line), and at $45^{\circ}$ (solid line). Due to the chromatic response of the OM, a coupling between $\varphi_{\text {sample }}$ and $\theta_{\text {sample }}$ appears in the systematic errors. The 
bias differs according to the strategy used for the sequential measurement. For the retardance $\phi_{E O}$ switching from $-\pi / 4$ to $\pi / 4$, the bias $\Delta \varphi_{\text {bias }}$ is uniform except for weak sample retardances. By contrast, for a switch with $\phi_{E O}$ from 0 to $\pi / 2$, the bias is low for a weak retardance but increases with the retardance for $\theta_{\text {sample }}$ at $45^{\circ}$ and no variation is observed for $\theta_{\text {sample }}$ at zero.

Figure 9(b) shows the bias on axis orientation for two different sample retardances, at 10 (solid line), and at 40 (dashed line). Once more, due to the chromatic response of the OM, a coupling between $\varphi_{\text {sample }}$ and $\theta_{\text {sample }}$ takes place, as shown by the systematic errors. The bias $\Delta \theta_{\text {bias }}$ is less significant for the configuration $\left(\phi_{E O}=0 \rightarrow \pi / 2\right)$.

3.2.2. Bias for slow set-up case: random phase $\varphi_{\text {rand }}$ not identical for the two sequential measurements. In this section we consider that during the two sequential measurements the random phase is not identical and fibres do not change their characteristics. Two sub-cases are distinguished: a retarder element in front of the sample or none at all.

No retarder element in front of the sample: The sample is a linear retarder with the retardance $\varphi_{\text {sample }}$ and the axis orientation $\theta_{\text {sample }}$. Biases on retardance and axis orientation are evaluated according to the values of $\varphi_{\text {sample }}, \theta_{\text {sample }}$ and the choice of the FWHM of the source spectrum between $50 \mathrm{~nm}$ (thick blue curves in figure 10) and $100 \mathrm{~nm}$ (thin red curves in figure 10). Figure 10(a) shows the bias on retardance for two different sample orientations, one at $0^{\circ}$ (dashed lines) and another at $45^{\circ}$ (solid lines). The coupling between $\varphi_{\text {sample }}$ and $\theta_{\text {sample }}$ is still present and amplified by the normalisation of the signal by the one defined at the surface. The larger the bandwidth, the larger the bias. Figure 10(b) shows the bias on axis orientation for two different sample retardances, of $10^{\circ}$ (solid lines), and of $40^{\circ}$ (dashed lines). Due to the need of employing the sample surface as a reference, it is necessary to switch $\phi_{E O}$ from $-\pi / 4$ to $\pi / 4$. With that, the bias on axis orientation is close to zero at $45^{\circ}$ and $135^{\circ}$ but increases for lower sample retardance and higher spectral width at $0^{\circ}, 90^{\circ}$ and $180^{\circ}$.

Retarder element in front of the sample: To be consistent with other retinal studies in the literature $[19,20]$, the retarder element corresponding to the cornea is chosen to have a one-pass retardance equal to $25^{\circ}$ and its axis orientation is set arbitrarily at $0^{\circ}$ on the basis $\left\{\left|e_{x}^{\prime}\right\rangle,\left|e_{y}^{\prime}\right\rangle\right\}$. The sample corresponding to the retinal nerve fibre layer (RNFL) has a one-pass retardance $\varphi_{\text {sample }}$ between $10^{\circ}$ and $50^{\circ}$ and is oriented at the angle $\theta_{\text {sample }}$. Biases on retardance and axis orientation are evaluated according to the values of $\varphi_{\text {sample }}$ and $\theta_{\text {sample }}$ (figure 10). Figure 11(a) shows the bias on retardance $\Delta \varphi_{\text {bias }}$ for two different sample orientations, at $0^{\circ}$ (dashed line) and at $45^{\circ}$ (solid line). Figure 11(b) shows the bias on axis orientation $\Delta \theta_{\text {bias }}$ for two different samples, the first one having a retardance of $10^{\circ}$ (dashed line), and the second one $50^{\circ}$ (solid line). In this sub-case, since the retardance $\phi_{E O}$ is switched from 0 to $\pi / 2$, the bias on axis orientation is weak (see figure $9(\mathrm{~b})$ ) and inferior to that obtained in figure 10(b). 


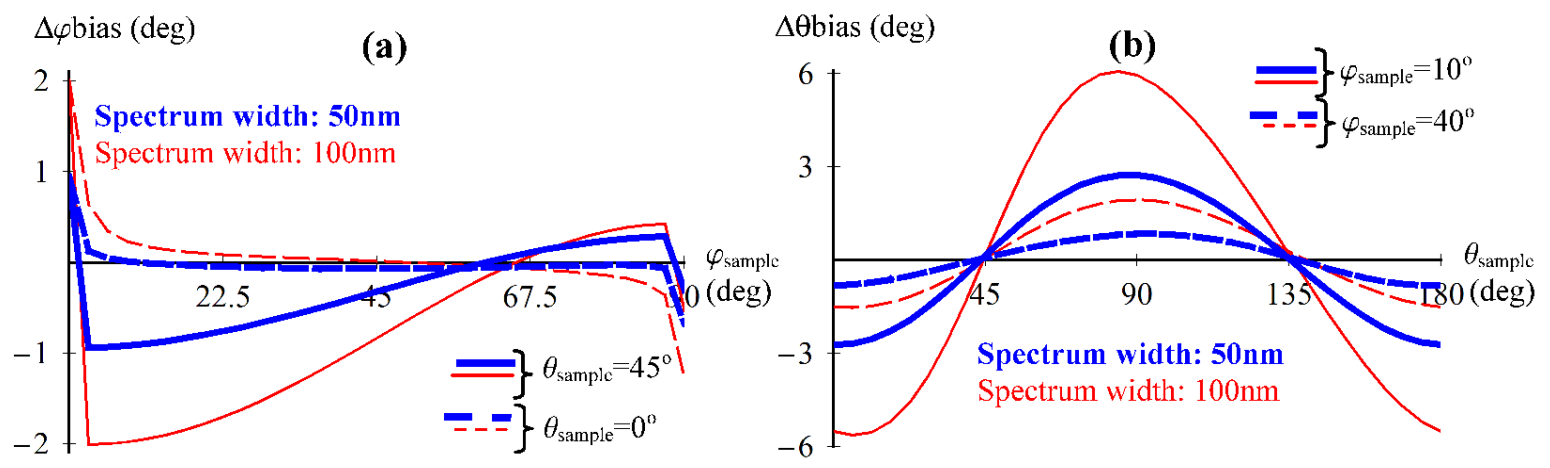

Figure 10. Measurement of the bias on the retardance $\varphi_{\text {sample }}$ (a) and on the orientation angle $\theta_{\text {sample }}(\mathrm{b})$ of a linear retarder. Thin curves: the FWHM of the source spectrum is equal to $100 \mathrm{~nm}$. Thick curves: the FWHM of the source spectrum is equal to $50 \mathrm{~nm}$. Solid lines in (a): sample oriented at $45^{\circ}$. Dashed lines in (a), sample oriented at $0^{\circ}$. Solid lines in (b): sample retardance equal to $10^{\circ}$. Dashed lines in (b), sample retardance equal to $40^{\circ}$.
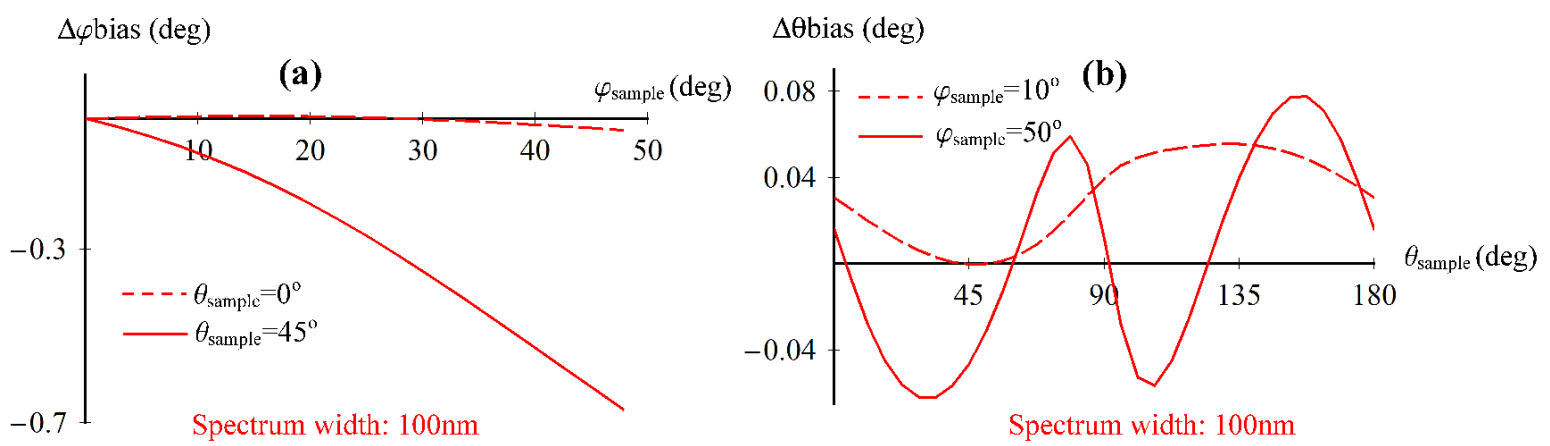

Figure 11. Measurement of the bias on the retardance $\varphi_{\text {sample }}(\mathrm{a})$ and on the orientation angle $\theta_{\text {sample }}(\mathrm{b})$ of a system made of a retarder (cornea) in front of a sample corresponding to the retinal nerve fibre layer (RNFL). Solid line in (a), sample oriented at $45^{\circ}$. Dashed line in (a), sample oriented at $0^{\circ}$. Solid line in (b), sample retardance equal to $50^{\circ}$. Dashed line in (b), sample retardance equal to $10^{\circ}$.

\section{Discussion and Conclusions}

The theoretical module presented requires some additional considerations regarding its practical implementation in an OCT system. The polarisation components within the optical module increase the optical path length of the sample arm, which demands a corresponding change in the optical path length of the reference arm. Moreover, inclusion of the $\mathrm{OM}$ in the system contributes to dispersion imbalance which needs to be compensated. Since perfect matching of dispersive properties of the different components in the reference arm would be difficult to achieve, we suggest the application of the Master-Slave Interferometry method [21], that makes the system tolerant to the unmatched dispersion.

As mentioned before, the linear polariser within the OM has two purposes: ensures 
that the light entering the sample arm is polarised, and performs the selection of the polarisation state on the return path. Accomplishing these roles inevitably involves losses; nevertheless, these roles are present in any PS-OCT system, leading to similar losses. If the source is partially polarised, one can minimise the input loss by placing a polarisation controller in front of the optical source to match the polarisation state of the light entering the sample arm with the axis of the linear polariser within the OM.

For practical implementation of the module there are two possibilities: (i) a freespace configuration which is not compact and can present some electric safety risk due to the high voltages required for the operation of the EO modulators and (ii) a fibrebased configuration which can be made more compact but will introduce additional implementation challenges. The new generation of ceramic EO modulators requiring lower driving currents [22] and compact Faraday rotators [23] allow for a fibre-based OM.

The theoretical model presented establishes a sequential procedure for employing a versatile optical module that, when placed within the sample arm of the interferometer, can provide the net retardance and axis orientation of the sample. Due to the dualpass nature of OCT systems, in a PS-OCT system it is not possible to measure any property other than linear retardance and linear diattenuation. Therefore, in this study, the only property being addressed is that of a linear retarder. The two sequential measurements require the acquisition of a channelled spectrum for each setting of the optical module. The procedures and optical module described ensure independence from potential internal disturbances created by the optical fibre, because the generation of the polarisation state (circular) and the analysis of the returned polarisation state are done in free space. The insensitivity of the system to fibre disturbances means that no calibration step is required in order to compensate for the polarimetric effects of the fibres. Distinction is made between short and long switching times of the EOs and equations are provided that establish the basis for the method presented, in respect to tolerance to phase disturbances when applied in a fast set-up and in a slow set-up. Simulations have been conducted to evaluate biases on retardance and axis orientation due to the chromatic response of the optical module. These biases act as systematic errors, relating to the choice of the spectral width. In any polarimetric measurement employing wide bandwidth sources, there is always a compromise to be made in terms of performance (accuracy of the retardance and axis orientation measurements) and bandwidth (axial resolution of the OCT system). Also, the accuracy requirement is dependent on the sample in question.

The particular case of a retarder in front of the sample is covered, which is applicable in polarimetric measurements of the retina disturbed by the anterior chamber of the eye.

Although the theory presented referred to the interrogation of the optical spectrum at the interferometer output, the module suggested can equally be used in time-domain OCT systems. 


\section{Acknowledgements}

S. Rivet acknowledges the Marie-Curie Intra-European Fellowship for Career Development, No. 625509. A. Bradu, M. J. Marques and A. Podoleanu were partially supported by the European Research Council (ERC) under the European Union's Seventh Framework Programme, Advanced Grant agreement "COGATIMABIO ", 249889. A. Podoleanu is also supported by the European Industrial Doctorate UBAPHODESA, FP7-PEOPLE-2013-ITN 607627, Royal Society Wolfson Research Merit Award and by the NIHR Biomedical Research Centre at Moorfields Eye Hospital NHS Foundation Trust and UCL Institute of Ophthalmology. M. J. Marques also acknowledges the University of Kent for his PhD support. The authors thank Sally Makin and Christopher Costa for proof-reading the manuscript.

\section{Appendix A. Jones matrices used in the theoretical model}

- Jones matrix of a polarisation rotator by an angle $\alpha$ in relation to either $\left|e_{x}\right\rangle$ or $\left|e_{x}^{\prime}\right\rangle$, depending on the case described in the text:

$$
J_{\operatorname{Rot}(\alpha)}=\left(\begin{array}{cc}
\cos \alpha & -\sin \alpha \\
\sin \alpha & \cos \alpha
\end{array}\right) .
$$

- Jones matrix for a linear birefringent medium with a retardance $\phi$, at an angle $\alpha$ in relation to either $\left|e_{x}\right\rangle$ or $\left|e_{x}^{\prime}\right\rangle$, depending on the case described in the text:

$$
J_{\operatorname{Bir}(\phi, \alpha)}=J_{\operatorname{Rot}(\alpha)} \cdot\left(\begin{array}{cc}
e^{i \phi / 2} & 0 \\
0 & e^{-i \phi / 2}
\end{array}\right) \cdot J_{\operatorname{Rot}(-\alpha)} \cdot
$$

The retardance introduced by a half-wave plate is $\phi=\pi$, and by a quarter-wave plate is $\phi=\pi / 2$.

- Jones matrix for a partial linear diattenuator with diattenuation $D$ and oriented at an angle $\alpha$ in relation to either $\left|e_{x}\right\rangle$ or $\left|e_{x}^{\prime}\right\rangle$, depending on the case described in the text:

$$
J_{\operatorname{Diat}(D, \alpha)}=J_{\operatorname{Rot}(\alpha)} \cdot\left(\begin{array}{cc}
\left(\frac{1-D}{1+D}\right) & 0 \\
0 & 1
\end{array}\right) \cdot J_{\operatorname{Rot}(-\alpha)} .
$$

\section{Appendix B. Demonstration of equation (6)}

In figure B1, the calculation of the field $\left|e_{b a c k}\right\rangle$ is detailed. This calculation comprises the polarimetric changes affecting the field $\left|e_{f w d}\right\rangle$. All the calculations have been carried out considering the point of view of the optical source; this means that the basis for $\left|e_{f w d}\right\rangle$ is given by $\left\{-\left|e_{x}\right\rangle,\left|e_{y}\right\rangle\right\}$.

$J_{\operatorname{Bir}(\phi, \alpha)}$ is defined in Appendix $\mathrm{A}$ as the generic Jones matrix of a birefringent medium considered as a linear retarder with retardance $\phi$, and oriented at an angle $\alpha$ 


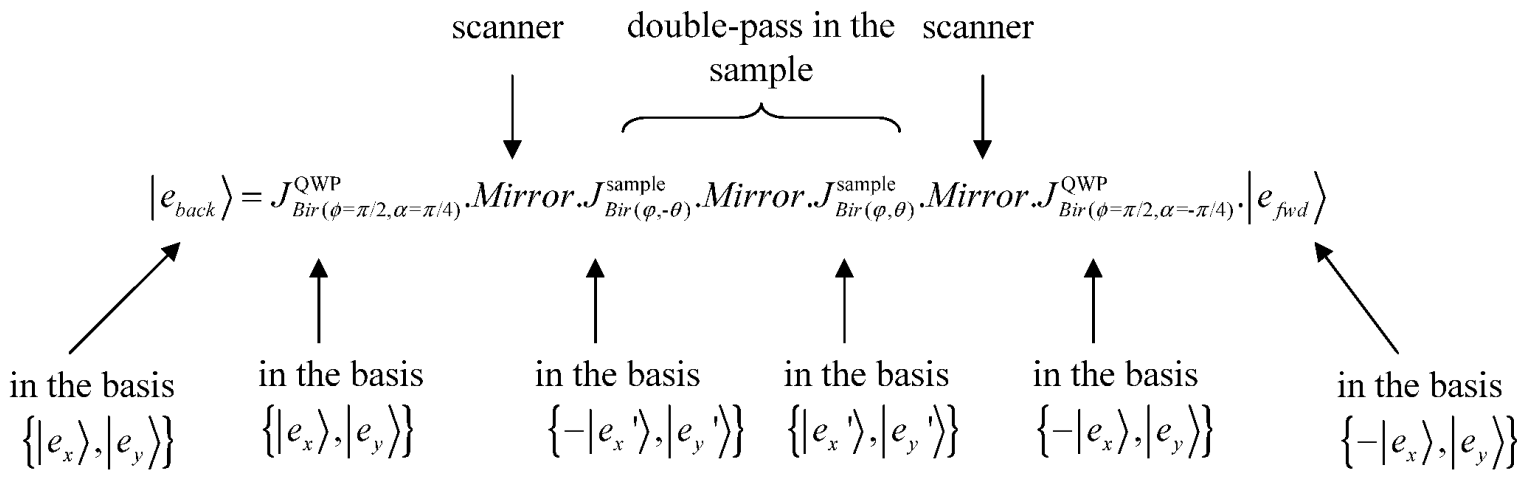

Figure B1. Calculation of the field $\left|e_{b a c k}\right\rangle$ defined in the basis $\left\{\left|e_{x}\right\rangle,\left|e_{y}\right\rangle\right\}$.

in relation to either $\left|e_{x}\right\rangle$ or $\left|e_{x}^{\prime}\right\rangle$, depending on the case described in the text. The Jones matrix for the mirror is given by

$$
\text { Mirror }=\left(\begin{array}{cc}
-1 & 0 \\
0 & 1
\end{array}\right)
$$

\section{References}

[1] Hee M R, Huang D, Swanson E A and Fujimoto J G 1992 J. Opt. Soc. Am. B 9903 ISSN 0740-3224

[2] Saxer C E, de Boer J F, Park B H, Zhao Y, Chen Z and Nelson J S 2000 Opt. Lett. 25 1355-1357

[3] Al-Qaisi M K and Akkin T 2008 Opt. Express 16 13032-13041 ISSN 1094-4087

[4] Zurauskas M and Podoleanu A 2013 J. Biomed. Opt. 18106010

[5] Braaf B, Vermeer K a, de Groot M, Vienola K V and de Boer J F 2014 Biomed. Opt. Express 5 2736 ISSN 2156-7085

[6] Yasuno Y, Makita S, Endo T, Itoh M, Yatagai T, Takahashi M, Katada C and Mutoh M 2004 Appl. Phys. Lett. 85 3023-3025 ISSN 00036951

[7] Baumann B, Choi W, Potsaid B, Huang D, Duker J S and Fujimoto J G 2012 Optics express 20 10229-10241

[8] Ding Z, Liang C P, Tang Q and Chen Y 2015 Biomed. Opt. Express 61828 ISSN 2156-7085

[9] Rashleigh S C 1980 Opt. Lett. 5392 ISSN 0146-9592

[10] Lippok N, Villiger M, Jun C and Bouma B E 2015 Opt. Lett. 40 2025-2028

[11] Trasischker W, Zotter S, Torzicky T, Baumann B, Haindl R, Pircher M and Hitzenberger C K 2014 Biomed. Opt. Express 52798 ISSN 2156-7085

[12] Roth J E, Kozak J A, Yazdanfar S, Rollins A M and Izatt J A 2001 Opt. Lett. 261069 ISSN 0146-9592

[13] Marques M J, Rivet S, Bradu A and Podoleanu A 2015 Opt. Lett. 40 3858-3861

[14] Götzinger E, Baumann B, Pircher M and Hitzenberger C K 2009 Opt. Express 17 22704-22717 ISSN 1094-4087

[15] Yamanari M, Makita S and Yasuno Y 2008 Opt. Express 16 5892-5906

[16] Zhang J, Guo S, Jung W, Nelson J and Chen Z 2003 Opt. Express 11 3262-3270

[17] Yamanari M, Makita S, Madjarova V D, Yatagai T and Yasuno Y 2006 Optics Express 146502 6515

[18] Barnes N P and Petway L B 1992 JOSA B 9 1912-1915

[19] Cense B, Chen T C, Park B H, Pierce M C and de Boer J F 2004 Journal of Biomedical Optics 9 $121-125$

[20] Knighton R W and Huang X R 2002 Investigative ophthalmology 6 visual science 43 82-86 
[21] Bradu A, Maria M and Podoleanu A G 2015 Optics express 23 14148-14161

[22] Jiang H, Zou Y, Chen Q, Li K, Zhang R, Wang Y, Ming H and Zheng Z 2005 Transparent electrooptic ceramics and devices Photonics Asia 2004 (International Society for Optics and Photonics) pp 380-394

[23] Shirasaki M, Kuwahara H and Obokata T 1981 Applied optics 20 2683-2687 